FORMATION Formation emploi

Revue française de sciences sociales

111 | Juillet-Septembre 2010

Pêle-mêle

\title{
Entre professionnalisation et salarisation, quelle reconnaissance du travail dans le monde associatif ?
}

Distinguishing between professionalising and paying a salary: recognising work in the non-profit world

Wie wird die Arbeit im Vereinswesen anerkannt- zwischen Professionalisierung und Vermehrung der Arbeitsverhältnisse

Entre profesionalización y salarización ¿qué reconocimiento del trabajo en el mundo asociativo?

\section{Annie Dussuet et Erika Flahault}

\section{OpenEdition}

Journals

Édition électronique

URL : http://journals.openedition.org/formationemploi/3091

DOI : 10.4000/formationemploi.3091

ISSN : 2107-0946

Éditeur

La Documentation française

Édition imprimée

Date de publication : 15 septembre 2010

Pagination : $35-50$

ISSN : 0759-6340

Référence électronique

Annie Dussuet et Erika Flahault, « Entre professionnalisation et salarisation, quelle reconnaissance du travail dans le monde associatif ? », Formation emploi [En ligne], 111 | Juillet-Septembre 2010, mis en ligne le 10 octobre 2012, consulté le 30 octobre 2020. URL : http://journals.openedition.org/ formationemploi/3091 ; DOI : https://doi.org/10.4000/formationemploi.3091 


\title{
Secteur d'activité
}

\section{Entre professionnalisation et salarisation,} quelle reconnaissance du travail
dans le monde associatif ?

Annie Dussuet, Erika Flahault*

\begin{abstract}
Dissocier les processus de salarisation et de professionnalisation permet de mieux analyser les conditions d'emploi dans le monde associatif. Cela permet aussi de comprendre comment les tentatives pour professionnaliser les activités associatives ne débouchent pas forcément sur une reconnaissance du travail effectué.
\end{abstract}

Le monde associatif n'est pas connu de prime abord pour son activité économique et son rôle d'employeur. Pourtant, même si le phénomène reste marginal à l'échelle des 1100000 associations actives aujourd'hui en France, l'emploi s'y est développé de façon importante dans les trente dernières années ${ }^{2}$. En 2005-2006, la part des associations employeuses est estimée à $16 \%$ par Viviane Tchernonog (2007); cela représente 172000 associations employant 1,9 million de salariés pour 1,05 million d'emplois équivalent

\footnotetext{
${ }^{1}$ Cet article est issu de plusieurs recherches empiriques portant sur les modes de gestion du travail dans les associations : une première version en a été présentée lors du colloque de clôture du programme «Économie sociale et solidaire : Acteurs, structures et dynamiques locales », financé par la région des Pays de la Loire (2006-2008); «Économie sociale et solidaire : nouvelles pratiques et dynamiques territoriales. Approches pluridisciplinaires », Nantes, 29-30 septembre 2008 .

${ }^{2}$ Notons que le fonctionnement sur une base uniquement bénévole et/ou militante est revendiqué par nombre d'associations.
}

* Annie Dussuet est maîtresse de conférences en sociologie à l'université de Nantes, CENS /Centre nantais de sociologie) et associée à l'équipe GTM (Genre, Travail et Mobilités) de I'UMR CRESPP (Unité mixte de recherche-Centre de recherches sociologiques et politiques de Paris). Ses recherches portent sur le travail ef l'emploi dans les secteurs féminisés des services, en particulier les services à domicile, sur la gestion des ressources humaines et la santé au travail dans les associations, dans une perspective de genre. Elle a publié récemment : Dussuet A. (2009), " De la «proximité » aux "services aux personnes »: le "privé » comme facteur d'inégalité entre les hommes et les femmes ", Économies et Sociétés, série Socio-économie du travail. Dussuet A., Noguès H. (2009), "Quel est l'impact des politiques sociales sur la santé des salariées intervenantes à domicile? ", Emploi et politiques sociales - Défis et avenir de la protection sociale, Barnay T., Legendre F. (dir.), 
Paris, L'Harmattan, pp. 29-41. Dussuet A. (2008), "Entre formel et informel, quelle place pour le travail dans les services aux familles développés par les associations? ", Les Mondes du Travail, n 5, pp. 81-94.

Erika Flahault est maîtresse de conférences en sociologie à l'université du Maine, ESO - Espaces et Sociétés UMR CNRS 6590. Ses recherches portent notamment sur les questions du travail et de l'emploi dans les associations (environnement et défense des droits des femmes), dans une perspective de rapports sociaux de sexe. Elle a publié récemment : Flahault E., Guardiola A. (2009), "Genre et associations en Europe : le pouvoir en question ", Informations sociales, $n^{\circ} 151$, janvier-février. "Genre et pouvoir en Europe », pp. 128-136. Flahault E., Loiseau D. (2009), "Que fait le salariat au militantisme dans les associations féministes? », @mnis, "Femmes et militantisme (Europe-Amérique, xIx siècle à nos jours) 》, http:// www.univ-brest.fr/amnis/documents/Loiseau2008.pdf. Flahault E. (dir.) (2006), L'insertion professionnelle des femmes. Entre contraintes et stratégies d'adaptation, PUR, coll. «Des sociétés 》, 266 p.

temps plein $(\mathrm{ETP})^{3}$. À côté du militantisme et du bénévolat $^{4}$, une part importante de l'action associative tend ainsi à s'appuyer sur du travail rémunéré.

Pour autant, les associations considèrent-elles ce travail de la même façon que les autres organisations productives, à but lucratif notamment ? Comment ces organisations économiques que sont assurément certaines associations, mais qui revendiquent souvent également une dimension politique, prennent-elles en compte le travail réalisé en leur sein ? Quelle reconnaissance lui octroient-elles ? Parviennent-elles à s'abstraire de leur environnement marchand pour accorder aux efforts accomplis et aux compétences déployées par leur ressource humaine, bénévole ou salariée, l'attention que les valeurs humanistes dont elles se réclament semblent nécessiter?

\footnotetext{
3 Soit plus ou moins l'équivalent du travail bénévole (935 000 ETP).

${ }^{4}$ Pour alléger la lecture, et parce que la question n'est pas au centre de notre propos dans le cadre de cette présentation, nous emploierons, dans le reste du texte, la notion de bénévolat pour évoquer toutes les formes d'engagement et de travail non accompagnées d'une rétribution salariale. Nous avons parfaitement conscience que d'un secteur à l'autre, d'une organisation à l'autre dans un même secteur, et même selon les individus dans une même association, les acteurs se définissent plutôt comme bénévoles ou comme militants, et que cela est loin d'être neutre du point de vue du sens du travail effectué.
}

Ces questions sont d'autant plus prégnantes que ces organisations sont aujourd'hui mises sur la sellette ; d'une part, par le monde économique et politique, qui n'hésite pas à stigmatiser les avantages concurrentiels dont leur statut non lucratif les ferait bénéficier, leur permettant ainsi de se satisfaire de performances économiques médiocres ; d'autre part, par les chercheurs, dont les travaux récents soulignent les dérives vers la précarisation de l'emploi auxquelles leurs pratiques en la matière peuvent conduire, comme en atteste la présence en leur sein de «près de $45 \%$ de formes d'emploi atypiques au sens $d u$ Code $d u$ travail » (Simonet, 2006). Les rémunérations versées par les organisations associatives apparaissent également plus faibles et la stabilité de l'emploi moins assurée (Legros, Narcy, 2004 ; Hély, 2007 \& 2009) que dans le reste de l'économie. Les associations sontelles donc simplement de « mauvais employeurs », ou bien utilisent-elles d'autres modalités que les autres organisations pour assurer la reconnaissance du travail?

Nous nous appuierons sur les recherches empiriques portant sur les modes de gestion du travail dans les associations ( $c f$. encadré 1). Nous montrerons ainsi que le caractère non lucratif de ces associations ainsi que la coexistence entre travail bénévole et travail salarié induisent certaines particularités dans la manière dont elles reconnaissent la contribution respective des travailleurs. Nous distinguerons ainsi la « salarisation », qui installe l'organisation dans la position d'employeur de salariés, de la « professionnalisation », avec laquelle elle est souvent confondue ; nous emploierons ici ce terme dans une acception plus restreinte en nous référant à la définition interactionniste (Hughes, 1996), comme processus de reconnaissance, par l'ensemble social, de l'exclusivité à exercer et de la légitimité à réguler l'activité dans un domaine déterminé. Nous montrerons que salarisation et professionnalisation ainsi entendues entraînent des formes a priori distinctes de reconnaissance du travail. Pour autant, dans les associations observées, ces formes se combinent pour déboucher sur des modalités originales. Nous proposerons une typologie de celles-ci en croisant les deux dimensions de la professionnalisation et de la qualification salariale ; cette dernière étant entendue ici comme la correspondance entre niveau de salaires et qualifications, référées elles-mêmes à 
des niveaux de formation. Nous soulignerons ainsi comment, dans certaines organisations associatives, l'accent mis sur la professionnalisation s'est opéré au détriment des modes de reconnaissance attachés au salariat, contribuant ainsi à sa déstabilisation dans la période contemporaine.

\section{LES ASSOCIATIONS ET LE TRAVAIL : SALARISATION VERSUS PROFESSIONNALISATION}

Le terme de professionnalisation est employé couramment comme un équivalent de celui de salarisation. Cet usage est le fait non seulement des acteurs associatifs, mais aussi des analystes qui interrogent le travail et l'emploi dans le monde associatif, s'alignant ainsi souvent sur le vocabulaire commun. Il s'agirait alors seulement de désigner le passage d'un travail bénévole, non rémunéré, à un travail qui, parce qu'il est rétribué par un salaire, serait d'emblée, et dans le même mouvement, reconnu comme « professionnel ». Cependant, cette superposition empêche d'analyser une dimension essentielle : celle de la reconnaissance sociale du travail. Nous proposons donc ici de distinguer nettement ces deux notions.

\section{La salarisation : une entrée dans l'univers marchand}

La salarisation peut en effet être définie par l'établissement d'un contrat de travail et par le paiement d'un salaire. Cependant, elle peut aussi, de facto, être définie comme l'entrée dans un univers marchand, dont les participants sont censés agir suivant leur propre intérêt, sans considération de celui d'autrui. Cela implique qu'à ce travail est appliquée une forme centrale de reconnaissance, d'ordre matériel, par la rémunération. Mais cela constitue aussi un paradoxe pour beaucoup d'associations qui inscrivent au contraire leur action dans une logique du don, le bénévolat en représentant la forme la plus caractéristique.

Toutefois, dans les sociétés occidentales, qu'on a pu qualifier de « sociétés salariales »(Castel, 1995), le salariat est largement balisé par le droit. La salarisa- tion signifie donc aussi l'entrée dans un système de règles qui définissent les modalités de reconnaissance du travail effectué.

Approchée à l'aune des critères objectifs, tels la durée des études nécessaires pour acquérir les savoirs et savoir-faire permettant la réalisation du travail, la « qualification » constitue la base d'un système de classification (Saglio, 1998) qui définit directement la rémunération. Mais elle est aussi, comme le souligne Pierre Naville, un « jugement social sur la qualité des travaux » (Naville, 1956).

Ainsi, la reconnaissance du travail dans le salariat se manifeste prioritairement dans l'ordre matériel de la rémunération. Toutefois, elle est aussi indissociablement, via la qualification, inscription dans une hiérarchie de l' " estime sociale » (Honneth, 2002); et ce, en référence à la contribution apportée à la réalisation des fins communes, dans une société globalement gouvernée par des principes marchands.

\section{La professionnalisation : une reconnaissance sociale au-delà du marché}

Quant à la notion de professionnalisation, dans la perspective que nous empruntons à la sociologie interactionniste américaine, elle se définit en premier lieu par la dimension de la reconnaissance. Pour Everett Hughes, "le concept de profession dans notre société n'est pas tant un terme descriptif qu'un jugement de valeur et de prestige. Il arrive très souvent que les gens qui exercent un métier tentent de modifier l'idée que s'en font leurs différents publics (...), leur propre conception d'eux-mêmes et de leur travail. Le modèle que ces métiers se donnent est celui de la "profession" » (Hughes, 1996).

Dans cette optique, la professionnalisation est un processus de construction sociale de la reconnaissance accordée à un groupe, qui devient par là même « professionnel ». Elle est achevée lorsque l'ensemble social concède à ce groupe une " licence » et un " mandat », c'est-à-dire " lorsqu'un groupe de gens s'est fait reconnaître la licence exclusive d'exercer certaines activités en échange d'argent, de biens ou de services. Ceux qui disposent de cette licence, s'ils ont le sens de la solidarité et de leur propre position, 


\section{Encadré 1}

\section{Le champ de la recherche : des associations employeuses}

Nous nous appuyons ici, d'une part, sur des recherches portant sur le travail et la gestion des ressources humaines dans les associations; d'autre part, sur les premiers résultats d'une recherche en cours sur la région des Pays de la Loire. :

Clergeau C., Dussuet A., Nogues H., Prouteau L., Schieb-Bienfait N., Urbain C. (2002), L'économie sociale et solidaire et les services à domicile aux personnes âgées, Rapport à la Préfecture de la région Pays de la Loire; Dussuet A., Loiseau D. (2007), "Les services aux familles offerts par les associations : un modèle de service " entre » formel et informel ", L'économie sociale entre informel et formel: paradoxes et innovations, Dussuet A., Lauzanas J.-M., Rennes, Presses universitaires de Rennes, coll. "Économie et société ", pp. 95-128; Flahault E., Robic P. (2007), "Les associations d'environnement partenaires privilégiés des pouvoirs publics : un paradoxe qui dynamise la gestion de l'environnement», L'économie sociale entre informel et formel. Paradoxes et innovations, A. Dussuet, J.-M. Lauzanas (dir.), Rennes, PUR, coll. "Économie et société », pp. 183-198; Dussuet A., Flahault E., Loiseau D. (2007), Quelle gestion des ressources humaines dans l'économie sociale? Entre bénévolat et professionnalisation : la place du travail dans les associations, Le Mans, Rapport à la Délégation interministérielle à l'innovation, à l'expérimentation sociale et à l'économie sociale (DIIESES).

Ces recherches nous ont amenées à étudier des secteurs associatifs extrêmement différents, tant par leur histoire que par leur dimension ou leurs rapports à la salarisation et à la professionnalisation. Notre attention s'est portée exclusivement sur des associations employeuses. Cependant, nous avons tenté d'appréhender le travail et les relations de travail dans toutes leurs dimensions, y compris bénévoles. Nous utilisons ici essentiellement des observations effectuées auprès d'organisations de services aux personnes, de protection de l'environnement et de défense des droits des femmes.

\section{Les associations de services aux personnes}

La plupart des associations de services aux personnes sont nées dans l'immédiat après-guerre : il s'agit alors, pour des militants de la mouvance catholique sociale, de promouvoir les milieux populaires et/ ou ruraux à travers l'entraide entre des familles éprouvées par des difficultés matérielles. Les premières salariées de ces associations sont les " travailleuses familiales 》, embauchées dès la fin des années 40. Leur mission consiste à suppléer les mères de famille.

Progressivement, ces associations étendent leurs activités en direction des personnes âgées ; puis, dans les années 70, en direction des enfants, avec les crèches parentales, en combinant des ressources bénévoles et salariées. Mais c'est à partir des années 80 que l'emploi salarié va se développer massivement dans les « services aux personnes ». II y a en effet concomitance d'une volonté politique de maintien à domicile des personnes âgées et d'une recherche de " gisements d'emploi » permettant de mettre au travail des chômeurs (qui sont en réalité ici des chômeuses).

De nombreux organismes vont alors se développer, soit directement issus des associations citées précédemment, soit avec des liens plus indirects, soit encore de façon autonome. Mais leur ancrage commun reste leur statut associatif, jusqu'aux années 2000 tout du moins. Les années récentes ont en effet vu la montée d'une concurrence du secteur lucratif. Ce dernier affirme sa légitimité à intervenir dans ce domaine en se prévalant de son efficacité économique. On assiste aussi à une diversification des services proposés : aide scolaire, accompagnement de déplacements, etc.

La comptabilisation des salariés du secteur apparaît difficile en raison du caractère atypique des emplois (temps partiels, emploi en "mandataire», multi-employeurs). Toutefois, pour 2004, la DARES (Direction de l'animation de la recherche, des études et de la statistique du ministère du Travail) compte, en France, 183000 salariés dans les organismes prestataires, dont la grande majorité sous statut associatif. 


\section{(Suite Encadré 1)}

Nous mobilisons ici des résultats issus de nos travaux de ces dix dernières années. Ils portent principalement sur des organisations associatives des départements de Loire-Atlantique et du Maine-et-Loire. Nous nous appuyons aussi sur des entretiens réalisés avec des responsables fédéraux en charge des questions de GRH (gestion des ressources humaines) au niveau national.

\section{Les associations de nature et d'environnement}

Les associations de nature et d'environnement sont très diverses, tant par leur taille, leur histoire, que leurs missions, leurs moyens d'action ou leurs territoires d'intervention. La grande majorité d'entre elles fonctionne aujourd'hui encore sur le seul engagement bénévole. Les premiers salariés y ont fait leur apparition dans les années 70 , mais leur nombre croit à la fin des années 90 . Un processus de professionnalisation est alors engagé. Ainsi, pour un tiers des structures employeuses, l'embauche du premier salarié remonte seulement à dix ans, alors même que les deux tiers d'entre elles ont plus de vingt ans d'existence.

Dans cette nébuleuse, il est mal aisé de dénombrer les emplois salariés. Toutefois, une étude réalisée par I'ANACT (Agence nationale pour l'amélioration des conditions de travail), en 2006, en collaboration avec la Fédération nationale des associations de nature et d'environnement (France Nature Environnement - FNE), forte de 3000 structures adhérentes, dresse le portrait suivant : un peu plus de $10 \%$ des associations enquêtées sont employeuses; un tiers d'entre elles compte 1 à 3 salariés et un quart plus de 9 . Ces salariés sont plutôt jeunes (45\% ont moins de 30 ans) et très qualifiés (50\% disposent d'un diplôme de niveau bac +3 ou au-delà, en rapport avec l'emploi occupé). Dans ce secteur en cours de professionnalisation, une quinzaine d'activités ont été répertoriées, notamment : l'éducation à l'environnement, l'expertise, la gestion de sites d'intérêt patrimonial, pour les activités du " cœur de métier », et la fonction d'assistant technique associatif pour les postes polyvalents, très répandus dans les associations à salarié unique.

Les exemples cités dans ce texte se fondent principalement sur le cas du réseau des Conservatoires d'Espaces Naturels (CEN), regroupés au sein de la Fédération Espaces Naturels de France (ENF), elle-même adhérente à FNE. L'ensemble du réseau, spécialisé dans la gestion concertée des sites d'intérêt patrimonial, recense 450 salariés dispersés sur tout le territoire français, dont 12 au sein de la fédération.

\section{Les associations de défense des droits des femmes}

La filiation est plus ou moins directe, mais toutes les associations de défense des droits des femmes sont indissociables du Mouvement de libération des femmes de la décennie 70. Parallèlement à l'analyse théorique de la place des femmes dans la société et la famille, elles ont développé une pratique de soutien aux femmes dans leur réalité quotidienne. Certaines associations ont débuté ces activités dans l'illégalité (contraception, avortement), et sans aucun subventionnement public. Elles ont ensuite obtenu la légalisation de ces activités et le financement, au moins partiel, de diverses manières, par des fonds publics nationaux, régionaux ou locaux.

Cette prise en charge de questions relevant de l'intérêt général a favorisé l'apparition et le développement de postes salariés. II s'agit souvent d'emplois aidés, en particulier pour l'information juridique (conseillères juridiques), l'accueil, l'accompagnement et/ou l'hébergement de femmes en difficulté (conseillères conjugales et familiales, conseillères en insertion professionnelle, assistantes sociales, monitrices éducatrices...) mais aussi plus largement pour la sensibilisation aux inégalités sexuées (formatrices, animatrices).

Parmi les associations défendant les droits des femmes, nous avons rencontré notamment des membres du Mouvement français pour le planning familial (MFPF), de Solidarités femmes (fédération des associations SOS femmes) et du Centre d'information sur les droits des femmes et des familles (CIDFF). 
revendiqueront un mandat pour définir les comportements que devraient adopter les autres personnes à l'égard de tout ce qui touche à leur travail (...) Quand la prétention d'un groupe à disposer d'un mandat d'une telle ampleur est reconnue comme légitime, explicitement ou implicitement, on peut considérer qu'une profession s'est établie » (Hughes, 1996). Cette reconnaissance est communément présentée comme la contrepartie de la renonciation, par le groupe professionnel, à tirer bénéfice de l'asymétrie d'information dont il dispose en raison de son savoir et de son savoirfaire ${ }^{5}$. Elle s'appuie sur la mise en évidence, par les professionnels, de leur capacité à s'extraire d'une perspective d'intérêt personnel pour promouvoir l'intérêt collectif. La mise en avant d'une éthique de la « profession » est donc indispensable au processus.

On peut ainsi remarquer que la professionnalisation réalise une entorse aux règles marchandes. Elle impose en effet des règles d'agrément pour l'exercice de l'activité faisant prévaloir son orientation vers un intérêt « social », sinon général, plutôt que vers des intérêts particuliers. Un travail «professionnel» se distingue donc des autres formes de travail : il implique la mise en œuvre de l'expertise détenue dans un domaine déterminé ; il suppose aussi, de la part du travailleur, une motivation non exclusivement instrumentale, qui fait aussi place à une mission tournée vers autrui ; il s'assortit enfin d'un contrôle de l'activité par l'ensemble des professionnels du champ.

Cette définition de la professionnalisation fait ainsi clairement apparaître la construction idéologique permettant la sanction sociale de la reconnaissance des compétences. De plus, cette acception de la profession comme activité orientée vers l'intérêt collectif ne nous paraît pas si éloignée des problématiques altruistes développées par les associations.

Pourtant, ce n'est pas tant la professionnalisation comme aboutissement que comme processus que nous souhaitons examiner dans le contexte associatif. D'autant que, d'une part, ce processus y apparaît souvent inachevé ou

\footnotetext{
${ }_{5}$ Ainsi, le médecin ou l'avocat pourrait profiter des information livrées dans le huis clos professionnel par le patient ou le client au détriment de celui-ci, ou bien encore faire payer son service un prix exorbitant lorsqu'il est seul détenteur des connaissances, médicales ou juridiques, requises face à une menace vitale. La coordination par le marché est alors insuffisante et l'appel à une déontologie professionnelle nécessaire.
}

partiel ; d'autre part, il s'y observe parfois en dehors de la relation salariale. Et à ce titre, les cinq dimensions du processus de professionnalisation déclinées par François Aballéa (2007) apportent un éclairage intéressant. Ainsi, au-delà de la dimension économique relative au marché sur lequel intervient le groupe professionnel et sous-entend «une fermeture relative de ce marché, qui peut aller jusqu'au monopole » (la licence de Hughes) et de la dimension institutionnelle qui renvoie à la définition des conditions d'exercice de l'activité (le mandat de Hughes), Aballéa identifie trois composantes complémentaires. La plus fondamentale réside dans la constitution d'une expertise (technique, sociale et gestionnaire) entendue comme définition et acquisition «d'un savoir propre qui distingue le professionnel du profane ». Elle implique le passage par un dispositif de formation plus ou moins formalisé. La dimension symbolique « correspond à la promotion d'une identité ». Elle assure un prestige et une reconnaissance particulière aux détenteurs du titre. Et enfin, la dimension culturelle « $s$ 'appuie sur la mise en place de dispositifs de socialisation (...) comme les institutions de formation professionnelle, les associations ou les syndicats professionnels ».

Rappelons aussi que la professionnalisation ne se construit jamais en référence au seul milieu associatif, même dans les cas où elle y est initiée. Bien au contraire, elle nécessite l'intervention d'acteurs extérieurs à ce milieu (secteur privé lucratif ou secteur public) et surtout, "dans tous les cas, les processus de professionnalisation s'appuient à un moment ou à un autre de leur déroulement sur l'action publique, c'està-dire qu'ils requièrent l'intervention de la puissance publique (...) qui valide l'espace de qualification en statuant sur les référentiels de formation et en certifiant les diplômes » (Aballéa, 2005).

Salarisation et professionnalisation sont donc deux processus distincts ; chacun d'eux relevant de logiques et de modalités diverses selon les contextes. Toutefois, dans une société comme la société française contemporaine, où les emplois salariés occupent $90 \%$ de la population active, ces deux processus apparaissent profondément enchevêtrés ; une reconnaissance de type " professionnel » s'opérant en effet également parfois pour certains emplois salariés (Voswinkel, 2007), grâce à l'intervention publique qui réglemente l'accès aux activités concernées. 
S'agissant des organisations associatives, nous nous intéresserons d'abord aux mouvements de salarisation qu'elles ont connus, avant d'examiner les processus de professionnalisation.

\section{DES PROCESSUS DIVERSIFIÉS DE SALARISATION}

Nos observations permettent de distinguer deux voies de salarisation : l'une comme conséquence logique du développement des activités de l'organisation ; l'autre comme élément constitutif du projet de l'association. Ces deux voies se distinguent non seulement par leur histoire et leur logique propre, mais aussi par l'attention portée à la qualité des emplois.

\section{L'emploi comme conséquence du développement associatif}

Dans la plupart des associations, le travail bénévole est premier. L'embauche de salariés n'intervient en effet que dans un second temps et d'une façon qui semble induite par les circonstances : les bénévoles s'épuisent face à une tâche qui s'accroît ; ils sont moins nombreux, ou bien encore, et ces motifs peuvent évidemment se cumuler, des opportunités de financement se présentent pour créer un poste salarié, destiné à seconder ou à remplacer un bénévole.

L'exemple type de ce mode de salarisation sur une conjonction de facteurs peut s'observer dans les organisations du secteur environnement. Il s'agit d'un moyen supplémentaire mis au service des buts de l'association. Dans ce secteur, les premiers permanents « salariés » font leur apparition dans les années 70, grâce à la mise à disposition des objecteurs de conscience ${ }^{6}$. Cette entrée par la petite porte, sous une forme proche d'un travail imposé, le service national, résulte de la rencontre des besoins des associations

\footnotetext{
${ }^{6}$ Le statut d'objecteur de conscience permettait aux jeunes gens refusant «pour des raisons de conscience » de faire usage des armes, de remplir les obligations du service militaire (ou service national). Le service de l'objecteur durait 20 mois (au lieu de 12) et s'effectuait dans le service civil d'une administration de l'État ou des collectivités locales ou encore dans un organisme à vocation sociale ou humanitaire. Depuis 1997, la conscription est supprimée en France, ce statut n'est donc plus en vigueur.
}

militantes, des idéaux et valeurs des militants objecteurs. Elle résulte aussi de l'opportunité, pour les pouvoirs publics, de «financer» des postes qui ne leur coûtent rien. Mais l'emploi salarié ne connaît un relatif essor, et dans une minorité de structures seulement, qu'à partir de 1997, avec la mise en place du dispositif « Nouveaux services-Emplois jeunes » ${ }^{7}$. Si l'emploi se développe si tardivement dans ce secteur, c'est qu'il ne constitue qu'une conséquence de l'évolution des structures, un moyen rationnel de mener à bien les missions environnementales qu'elles se donnent ; en même temps, l'emploi est une condition de la poursuite du développement de l'activité, face à une population bénévole stabilisée mais ne progressant plus. On retrouve ainsi l'application d'un principe affirmé par les institutions représentatives du secteur associatif, comme la CPCA (Conférence permanente des coordinations associatives) : « le projet doit rester premier... ». Les résultats d'une enquête menée pour le Fonjep (Fonds de coopération de la Jeunesse et de l'Éducation populaire) concluent que « si le projet ne mène pas nécessairement à l'emploi, l'emploi découle obligatoirement du projet, même s'il peut avoir pour but, à un moment donné de la vie de l'association, plutôt dans une phase de démarrage, d'aider à le préciser et à l'enrichir» (2003).

\section{L'emploi comme partie intégrante du projet associatif}

Dans d'autres cas, c'est le travail lui-même qui impose le recours à des salariés, souvent dès la création de l'association : soit parce que des compétences spécifiques que les bénévoles ne possèdent pas apparaissent nécessaires ou sont imposées par la réglementation ; soit parce que les conditions de réalisation du travail (pénibilité, horaires, déplacements) ne correspondent pas à leur définition de l'action bénévole.

\footnotetext{
${ }^{7}$ C'est également en 1997 que le dispositif « Nouveaux services - Emplois jeunes » est mis en place par le Plan national pour l'emploi et de lutte contre le chômage. Il a pour double objectif de développer des activités d'utilité sociale répondant aux besoins émergents ou non satisfaits, et de favoriser l'insertion professionnelle durable des jeunes. Le contrat d'embauche, conclu entre le jeune et un employeur du secteur public ou un organisme à but non lucratif, est un contrat ordinaire de droit privé, à l'exception de la durée du contrat à durée déterminée qui, en l'espèce, est de cinq ans. Depuis fin 2002, il n'est plus possible de créer de nouveaux postes emplois-jeunes. En revanche, les contrats en cours à cette date se sont poursuivis jusqu'à leur terme, en bénéficiant de l'aide de l'État.
} 
C'est par exemple le cas dans le secteur des services à domicile. Dès les années 40 , des associations familiales s'engagent dans une activité d'aide aux mères de famille ; cela suppose de se déplacer pour intervenir dans les foyers à des horaires où les «ménagères militantes » (Loiseau, 1996) sont elles-mêmes occupées par leurs propres tâches domestiques. Il s'agit aussi de réaliser des tâches qui, pour banales qu'elles soient, apparaissent comme suffisamment fondamentales, dans une perspective familialiste (Lenoir, 2003), pour constituer un véritable « travail ». Fournir ce service suppose donc l'embauche de jeunes femmes qui, issues des milieux populaires, n'ont d'autres moyens d'existence que ceux procurés par leur travail. Ces organisations associatives sont souvent proches du mouvement ouvrier. Elles sont donc soucieuses de respecter les règles de reconnaissance du travail, obtenues par la lutte syndicale; dès lors, elles ne peuvent envisager de proposer à ces jeunes femmes une simple occupation bénévole. Les valeurs dont les organisations sont porteuses impliquent de rémunérer le travail accompli ; mieux, de lui accorder une véritable reconnaissance à travers des conditions d'emploi satisfaisantes eu égard aux normes en vigueur. La création d'emplois salariés est alors d'emblée une évidence.

\section{Des conséquences en termes de qualité des emplois}

L'appartenance à un cas ou à l'autre dépend non seulement du secteur mais aussi et surtout du type d'association (missions, histoire, contexte d'émergence...) et de la position dans le cycle de vie du secteur luimême. Ainsi, dans un même réseau associatif, les premières organisations mises en place ont pu fonctionner pendant un temps sur le seul bénévolat. À l'inverse, les structures nées plus récemment ont d'emblée accueilli des salariés et bénéficié des expériences des premières. On peut ici citer l'exemple du réseau des CIDFF (Centre d'information sur les droits des femmes et des familles). Les premières associations, créées au début des années 70, reposaient uniquement sur le bénévolat; celles qui naissent dans les années 80 ont immédiatement recours au travail salarié.

Surtout, les conséquences de ce positionnement se manifestent directement sur les conditions d'emploi des salariés. Dans cette logique de salarisation simple, les organisations se contentent de reprendre, dans l'emploi associatif, les catégories utilisées ailleurs par le monde social : les « qualifications », reconnues ou pas, aux salariés associatifs, sont, au mieux, celles construites dans les négociations avec le patronat classique et qui fondent les grilles de classification. La question de la reconnaissance du niveau de formation ou des compétences mises en œuvre est alors en quelque sorte internalisée dans le rapport salarial ; elle est en effet rabattue sur les aspects matériels définis par le contrat de travail, et en tout premier lieu sur la qualification.

Mais nombre de structures associatives sont hors convention collective ou adhèrent à une convention collective adoptée «par défaut », en l'absence de négociations propres à leur activité spécifique. Dans ce contexte, et quand l'emploi est envisagé essentiellement comme un coût, la proximité salariat/bénévolat et la substituabilité du travail salarié au travail bénévole constituent souvent un risque de dénégation de la qualification : si les bénévoles mettent en œuvre gratuitement les mêmes compétences que les salariés, une rémunération élevée de ces derniers semble inutile, voire injuste. En effet, cela semble signifier que «n'importe qui », avec « un peu de bonne volonté », peut effectuer le travail. Par exemple, dans le secteur des services à domicile, la fonction de « responsable de secteur », chargée de l'organisation des plannings et de l'encadrement de proximité des intervenantes à domicile, a longtemps été exercée par des bénévoles. Dès lors, elle peine à obtenir une reconnaissance en termes de qualification. Certaines organisations considèrent ces responsables de secteur comme de « simples secrétaires ». Ce risque s'accroît dans les cas où l'emploi salarié découle du développement associatif ; d'autant plus que les employeurs restent des bénévoles ${ }^{8}$, issus d'horizons très différents et souvent éloignés de la gestion des ressources humaines. Ils éprouvent des difficultés à intégrer pleinement cette « fonction employeur », nouvelle pour eux et pour l'organisation. Les conditions de travail, le niveau des rémunérations, les droits reconnus aux salariés peuvent alors être l'objet de disparités importantes au sein d'un même réseau associatif, selon la sensibilité ou la combativité des employeurs comme des salariés vis-à-vis de ces questions.

${ }^{8}$ Rappelons que dans les associations, l'employeur reste le Conseil d'administration (CA), uniquement composé de bénévoles. 
Dans certains cas, la question de la qualification, intrinsèquement liée au contrat salarial, s'est ainsi trouvée purement et simplement éludée ou encore traitée de façon très hétérogène d'une structure à l'autre ; en effet, soit la nouveauté de l'activité ne permettait pas l'inscription dans une grille de classification, soit certaines associations jugeaient primordial de limiter leurs coûts alors que d'autres prenaient davantage conscience de leur responsabilité d'employeur.

Mais surtout ces modalités différentes de salarisation se distinguent par leur rapport, de substituabilité ou de complémentarité, au travail bénévole. Elles vont se traduire aussi par une imbrication plus ou moins forte dans les processus de professionnalisation.

\section{UNE PROFESSIONNALISATION IMBRIQUÉE DANS LA SALARISATION}

Dans les différents secteurs d'activité considérés, ces processus de salarisation ne sont pas exclusifs de mouvements de professionnalisation. Ceux-ci s'imbriquent de diverses manières, salarisation et professionnalisation se cumulant, se renforçant mutuellement parfois, ou bien encore s'excluant l'une l'autre.

\section{De la «professionnalisation » des salariés...}

Parmi les associations observées, certaines ont tenté une professionnalisation de leurs salariés. Dans plusieurs cas, elles ont véritablement innové en " inventant » en quelque sorte des activités nouvelles, ou plus modestement de nouvelles manières de les effectuer. L'intervention associative a alors consisté à transformer une activité auparavant réalisée sur un mode informel, qu'il soit bénévole, militant ou domestique, en un travail, salarié le plus souvent mais pas toujours, sans accepter que la salarisation en transforme le contenu, en contrôlant donc les manières de l'effectuer. Pour les organisations, cela nécessite d'obtenir à la fois licence et mandat, caractéristiques de la professionnalisation.

La reconnaissance, par les pouvoirs publics, de la nécessité d'une formation spécifique pour exercer l'activité, quel qu'en soit le niveau scolaire, en constitue un élément essentiel. Les associations sont plus ou moins bien parvenues à l'obtenir.

Dans le cas des travailleuses familiales, par exemple, il s'agissait de faire réaliser, sur un mode salarié, des tâches habituellement accomplies gratuitement par les mères de famille, dans une logique de don. Le risque étant, en faisant apparaître la dimension marchande $\mathrm{du}$ travail, d'induire un soupçon sur les motivations de la salariée, empêchant par là même son immixtion dans l'intimité des logements privés. Les deux modèles de services apportés jusque-là à domicile par des salariées, celui des domestiques, d'une part, celui des assistantes sociales d'autre part, étaient aussi inacceptables l'un que l'autre dans ces foyers ouvriers modestes, bien que pour des raisons opposées. La travailleuse familiale ne devait être ni une « bonne », emblématique d'une posture de soumission, ni une « bourgeoise », perçue comme vecteur de contrôle social à travers des manières de faire étrangères au milieu. Cela obligeait à " inventer »une modalité nouvelle de l'activité salariée tenant compte de ces contraintes. La formation, obligatoirement suivie par les postulantes travailleuses familiales, intégrera ainsi une dimension novatrice d'alternance entre formation institutionnelle et stage permettant une socialisation au milieu d'exercice.

L'objectif des informatrices juridiques du CIDFF était de mettre à disposition d'un public, d'abord féminin, des informations sur le droit lui permettant de prendre en main sa situation. Cela supposait des connaissances juridiques attestées mais aussi une bonne connaissance de la genèse et des mécanismes des inégalités de genre. Il s'agissait de distinguer, dans l'ensemble des textes de droit, les éléments susceptibles d'être utilisés dans une optique égalitaire. C'était donc là une modalité nouvelle d'une activité de type professionnel, existante par ailleurs, qui restait à inventer.

Certaines organisations ont dans le même temps cherché à obtenir une reconnaissance matérielle de ces activités nouvelles, sur le mode salarial. Elles ont ainsi réalisé une action de lobbying auprès des pouvoirs publics. La reconnaissance professionnelle s'institutionnalise en effet à travers la réglementation et le financement par les organismes de protection sociale ou les services de l'État; notamment par 
les qualifications, les titres professionnels, les agréments ou encore l'encadrement du marché. Selon les contextes économiques, politiques et sociaux, les organisations ont été plus ou moins entendues.

Ainsi les travailleuses familiales constituent un exemple de professionnalisation plutôt réussie ; en effet, dès 1949, une sorte de « licence » leur est délivrée. Est ainsi instaurée une obligation de formation (par des organismes issus du monde associatif) pour les interventions financées (partiellement) par les Caisses d'allocations familiales (CAF) ${ }^{9}$. Ici, ce sont les associations qui ont insisté sur la nécessaire formation, notamment pour prémunir les femmes contre les risques de ce travail de suppléance des épouses. Mais cette reconnaissance professionnelle intervient dans une période favorable à tous égards. D'une part, le pouvoir politique est relativement proche des associations en question ; d'autre part, la Sécurité Sociale vient d'être créée, avec sa branche famille ; et enfin, les tâches domestiques réalisées par les mères de familles, souvent nombreuses, sont suffisamment importantes pour légitimer l'intervention de « professionnelles ». Cette reconnaissance professionnelle s'est également traduite par la signature, en 1957, d'une convention collective. Elle inscrit le certificat de travailleuse familiale comme qualification reconnue dans une grille de classification et nécessaire pour occuper l'emploi.

En revanche, dans le même secteur, les organisations associatives employeuses ont cherché, depuis les années 60 , à améliorer l'image des aides à domicile qui interviennent auprès de personnes âgées, en les distinguant de la domesticité. Mais ce n'est qu'en 2002 qu'un accord de branche est intervenu. Il distinguait des degrés de complexité des interventions en les liant strictement à des niveaux de diplôme détenus ; il devait ainsi permettre aux salariées diplômées d'accéder à un niveau de rémunération substantiellement plus élevé que celui des salariées non formées (Clergeau, Dussuet, 2005). Mais les mesures du plan Borloo de développement des services aux personnes ${ }^{10}$

\footnotetext{
9 Les associations employeuses obtiennent également au passage une sorte de « mandat » en raison de la délégation de diagnostic qui leur est concédée par les Caisses.

${ }^{10}$ Dans une perspective de création d'emploi, le Plan Borloo (2005) a englobé, dans la définition des « services aux personnes » ouvrant aux déductions fiscales, un ensemble des services allant du ménage au conseil personnalisé en informatique en passant par le
}

ont mis à mal cette reconnaissance. Elles ont en effet brouillé à nouveau les frontières entre services d'aide aux personnes fragiles et services domestiques (JanyCatrice, Puissant, Ribault, 2009).

À l'inverse, l'activité des informatrices juridiques du CIDFF n'est pas réglementée. Les salariées engagées sont pourtant généralement titulaires d'une formation de niveau master. Mais il ne s'agit là que du résultat des politiques associatives de recrutement et de formation. Elles ne s'assortissent pas d'une politique salariale correspondante. Seules les associations employeuses sont donc garantes de leur compétence. Les incertitudes qui pèsent sur elles concernant leur financement ne les engagent pas à élever leurs exigences, ni le niveau des rémunérations, qui reste d'autant plus bas que le temps partiel y est la règle. Sans doute, l'activité est plus récente. Cependant, on peut aussi penser que les objectifs d'information sur les droits des femmes de ces associations apparaissent moins prioritaires aux pouvoirs publics dans le contexte contemporain.

Dans le secteur de la gestion des espaces naturels, les nouveaux métiers environnementaux, au cœur d'un processus de professionnalisation toujours en cours, font rapidement l'objet d'une réflexion en termes de qualification et de formation. En effet, les structures se sont saisies de l'opportunité offerte par le dispositif « Nouveaux services-Emplois jeunes ». D'une part, pour créer des emplois ; d'autre part, pour construire un secteur d'activité à part entière, reconnu en tant que tel. Mais en l'absence de monopole des structures associatives sur ces emplois ${ }^{11}$, la question prend une autre dimension. L'élaboration d'une qualification commune, reconnue par tous les acteurs du champ, passe alors d'un côté, par une mise à plat des référentiels métiers développés, dans un premier temps, dans chaque type de structure isolément, et de l'autre, par la réalisation de référentiels métier communs qui définissent les métiers, les missions, les responsabilités, l'autonomie et les compétences initiales requises. Elle dépend aussi d'une réflexion, en cours, sur les questions d'identité professionnelle. L'objectif à court

coaching, les cours particuliers à domicile et l'accompagnement de personnes âgées.

${ }^{11}$ La plupart des nouveaux métiers environnementaux se développent conjointement au sein du secteur associatif, des collectivités locales et des entreprises privées. 
terme étant d'une part, de faire entrer ces nouveaux métiers dans le ROME, Répertoire opérationnel des Métiers et des Emplois de l'ANPE (Agence nationale pour l'emploi devenue Pôle Emploi depuis décembre 2008, suite à la fusion avec les Assedic) et d'autre part, de participer à l'organisation de l'offre de formation; offre qui se développe de façon foisonnante et souvent déconnectée des besoins réels des structures. Mais, au-delà des démarches politiques menées principalement par les instances fédératives au niveau local, les besoins en formation ne sont pas toujours bien identifiés. Et si les administrateurs se déclarent sensibles à la professionnalisation de leurs salariés, ils sont finalement peu porteurs d'initiatives. Les salariés, acteurs de leur professionnalisation, sont souvent les initiateurs de leurs formations. En outre, ces difficultés à identifier, voire à penser, les besoins en formation présentent une acuité particulière pour les salariés gestionnaires et d'encadrement. Ce qui peut paraître paradoxal dans la mesure où ces personnels ont souvent été recrutés dans le giron des associations, au sein des bénévoles, sans aucune qualification initiale en rapport avec les fonctions à assumer. La prise de conscience se diffuse pourtant progressivement des niveaux fédératifs aux niveaux locaux.

Les associations observées s'attachent donc à professionnaliser leurs salariés, mais à travers des processus divers ne se traduisant pas nécessairement par une reconnaissance d'ordre matériel.

\section{... à la « professionnalisation » des bénévoles}

Par ailleurs, si les processus de salarisation et de professionnalisation sont souvent liés, ils peuvent aussi être disjoints : on assiste ainsi parfois à la recherche d'une professionnalisation des bénévoles.

Dans l'aide à domicile, les responsables de secteur, qui ont pour fonction d'organiser le travail des intervenantes, ont longtemps été des bénévoles. Mais depuis quinze ans environ, la complexité de leur tâche devient telle ${ }^{12}$ que beaucoup d'associations ont embauché des

\footnotetext{
12 Il s'agit en effet non seulement d'établir des plannings d'intervention de plus en plus contraints par les exigences des financeurs, mais aussi d'effectuer une véritable gestion des ressources humaines en ajustant les contrats de travail des salariées à ces plannings, dans le respect du droit du travail, et en tenant compte de la pénibilité physique et psychique des tâches à réaliser.
}

salariées pour l'effectuer. Certaines continuent néanmoins à défendre un modèle dans lequel les bénévoles occupent ces postes; et ce au nom du lien social qu'ils sont censés établir dans cette position d'intermédiation entre les intervenantes salariées et les utilisateurs du service. Cela implique, de la part des organisations, un important effort de formation de ces bénévoles... qui deviennent de cette manière des professionnels, mais non salariés. Pourtant, on peut douter de l'achèvement de ce processus ; en effet, cette reconnaissance de professionnalité reste interne aux organisations, largement invisible donc pour la société environnante. Cela peut même constituer un obstacle à la reconnaissance professionnelle des salariées qui occupent ce type de poste.

Dans le secteur des droits des femmes, les bénévoles du Planning Familial sont, jusqu'à très récemment, soumises à la même obligation que les salariées de suivre une formation de $450 \mathrm{~h}$ en conseil conjugal et familial. Cette formation est alors perçue comme garante du professionnalisme, et comme facteur d'homogénéisation, structurant à la fois le " potentiel d'expertise » et l'association. Depuis quelques années, les bénévoles sont moins directement présentes auprès des publics accueillis ; cependant, toutes les bénévoles participant à l'accueil ont suivi la formation en question.

Ces exemples révèlent combien la réussite $\mathrm{du}$ processus de professionnalisation enclenché par les associations, et le plus souvent intimement lié à la salarisation, dépend de la capacité de ces organisations à faire reconnaître leur légitimité à définir le service qu'elles offrent.

La reconnaissance sociale s'appuie alors sur la spécificité des compétences nécessaires à une activité associative irréalisable par des travailleurs ne partageant pas les valeurs portées par les organisations. Mais elle ne peut être complète que si ces dernières s'engagent dans un processus social d'élaboration de la reconnaissance ; ce processus se déployant à de multiples niveaux, du politique au médiatique, et visant à définir une représentation sociale commune valorisante du travail effectué. Lorsque cet objectif est atteint, il se traduit, dans la société contemporaine qui reste structurée autour du salariat, par la reconnaissance d'une qualification attestant des compétences mises en œuvre. Cette qualification se traduit ellemême par l'amélioration des conditions d'emploi, de la rémunération tout particulièrement. 
Tableau 1

Quatre types de travailleurs associatifs

\begin{tabular}{|c|c|c|}
\hline & $\begin{array}{l}\text { Professionnalisation } \\
\text { associative - }\end{array}$ & $\begin{array}{c}\text { Professionnalisation } \\
\text { associative + }\end{array}$ \\
\hline Qualification salariale - & Travailleur abstrait & Professionnel méconnu \\
\hline Qualification salariale + & Salarié professionnel & Professionnel associatif salarié \\
\hline
\end{tabular}

\section{QUELS MODES}

DE RECONNAISSANCE POUR LES TRAVAILLEURS ASSOCIATIFS?

À des moments de leur existence, certaines associations apparaissent donc plutôt orientées vers une logique de salarisation " simple»; à d'autres périodes, ou dans d'autres organisations, la logique de professionnalisation prédomine, adressée le plus souvent au travail salarié, mais aussi parfois, on l'a vu, au travail bénévole. En articulant ces deux dimensions de professionnalisation et de qualification salariale, on peut ébaucher une typologie des travailleurs associatifs. Elle croise ces deux modalités de reconnaissance, d'ordre salarial ou professionnel. Elle est construite empiriquement à partir d'observations d'ordre monographique dans des secteurs bien définis. Cependant, elle nous semble pouvoir être généralisée à d'autres secteurs de l'économie sociale ${ }^{13}$. Elle présente en outre l'intérêt de faire apparaître plus clairement le rôle joué par les associations dans un contexte général de précarisation de l'emploi salarié.

On peut ainsi distinguer quatre types ${ }^{14}$ de travailleurs associatifs, suivant le degré plus ou moins important d'implication des associations employeuses dans le processus de professionnalisation ${ }^{15}$ (professionnali-

13 À condition d'exclure les emplois développés par les associations d'insertion qui nous paraissent relever d'autres logiques. En effet, l'objectif de ces associations est de permettre l'emploi ou le retour à l'emploi de personnes qui en sont éloignées, et pour cela elles font un usage récurrent des contrats aidés, par définition et par mission dans ce cas précis, à durée déterminée. De ce fait, elles occupent une position à part au regard de l'emploi.

${ }^{14}$ Soulignons qu'il s'agit là d'une construction idéale de types « purs » qui ne trouvent jamais de réalisation complète, et entre lesquels existe évidemment une infinité de situations intermédiaires. L'observation du monde réel ne permet donc qu'un classement toujours incertain et discutable.

15 Cette implication ne préjuge pas du degré d'aboutissement du processus, ni de la présence d'autres acteurs, éventuellement sation associative), et celui de la reconnaissance de qualification ${ }^{16}$ (qualification salariale) ( $c f$. tableau 1) :

- le « travailleur abstrait 》 réalise, dans l'association, des tâches dont la qualification n'est pas ou guère reconnue ; ce travailleur pourrait être, au fond, n'importe qui : à l'image du prolétaire du XIX siècle, il ne fournit que du " travail humain abstrait ${ }^{17}$ » (Marx, 1975). Seul importe le résultat de son action, ses qualités personnelles ne sont donc pas en jeu. Il peut, et c'est le plus souvent le cas, être salarié. Mais un bénévole peut également exécuter ces tâches, même si aucune référence aux "valeurs " portées par l'association ne leur est associée. D'une part, aucune reconnaissance de qualification n'est attachée à ce travail dans l'ordre salarial ; d'autre part, comme l'association ne revendique pour lui aucune spécificité, aucun processus de professionnalisation n'est engagé. Ce travailleur est ainsi, somme toute, " abstrait ». Par exemple, la personne qui réalise bénévolement de la mise sous enveloppe ne mobilise pas une compétence reconnue, ni dans le cadre associatif, ni dans un autre contexte ; de même pour le personnel, en général salarié, chargé de l'entretien des locaux. Il s'agit pourtant bien d'un travail, au sens d'une dépense d'énergie. Mais il n'est ni qualifié, ni professionnel. La reconnaissance est ici la plus faible.

d'autres employeurs non associatifs, dans ce processus. Une activité peut être très peu avancée dans un processus de professionnalisation, bien que les associations employeuses déploient en sa faveur une énergie importante. À l'inverse, les associations peuvent n'avoir aucune part dans un processus qui est cependant très avancé, voire abouti.

${ }^{16}$ Cette reconnaissance de qualification peut avoir été obtenue grâce à l'action des associations, ou bien en être totalement indépendante.

17 Dans son analyse de la marchandise, Marx explique par exemple que « le tissage ne se distingue en rien de la taille des vêtements, c'est-à-dire est du travail humain abstrait » (Marx, 1975, p. 65) : peu importe donc le travail concret réalisé, dès lors qu'il apporte de la valeur à la marchandise. De même ici, un travailleur ne se distingue pas d'un autre par ses qualités personnelles. 
- le « salarié professionnel » dispose d'une reconnaissance de sa qualification en dehors du milieu associatif, dans les institutions du monde salarié, à travers la réglementation, les conventions collectives et les grilles de classification. C'est aussi à l'extérieur du monde associatif qu'un processus de professionnalisation a pu se réaliser, plus ou moins reconnu au niveau salarial.

C'est le cas du médecin gynécologue qui assure des permanences dans les centres de planification du Planning familial. Sa qualification est reconnue en dehors de l'organisation. Il n'y réalise de toute façon qu'une part de son activité, la part principale s'effectuant en libéral ou en milieu hospitalier. C'est le cas aussi du comptable qui mobilise, au service de l'association, une qualification professionnelle qui ne doit rien au monde associatif et dont la mise en œuvre peut tout à fait se passer de référence aux valeurs de l'organisation associative dans laquelle elle s'inscrit. C'est en tant que spécialistes reconnus que l'un et l'autre interviennent, mais cette reconnaissance s'est construite et s'actualise en dehors du monde associatif.

- le « professionnel associatif salarié » exerce, au contraire, un métier qui a été « inventé » par les associations et pour lequel elles ont également obtenu une reconnaissance de qualification valant « licence » pour exercer. Le processus de professionnalisation est ici à l'initiative des associations. Son aboutissement est tributaire de leur activisme, mais il a franchi les frontières du monde associatif. Le « professionnel associatif salarié » a vu sa professionnalisé portée par le monde associatif, mais elle est à présent reconnue au-delà.

Les travailleuses familiales que nous avons déjà évoquées en sont un bon exemple. Les gestionnaires de sites naturels salariés par les associations naturalistes constituent un cas un peu différent. Cette activité a commencé à se développer conjointement dans les associations (conservatoires régionaux d'espaces naturels) et dans le secteur public (parcs nationaux). Elle est entrée dans un processus de professionnalisation depuis le milieu des années 90. Celui-ci est porté tant par les acteurs publics que par les acteurs associatifs. Ils y ont déployé une énergie considérable pour participer aux négociations sur les référentiels métier et formation, pour s'inscrire dans la réflexion en cours sur l'identité professionnelle attachée à ces nouveaux métiers et pour investir les instances de socialisation professionnelles que sont les réseaux nationaux. Et en premier lieu, le GIP Aten ${ }^{18}$, fondé sur le principe de la mutualisation des moyens et des compétences. L'une de ses missions essentielles consiste justement à contribuer à l'émergence et à la reconnaissance d'une filière professionnelle.

Dans ce cas, la reconnaissance sur le mode salarial se cumule avec une reconnaissance spécifique au milieu associatif. Elle offre au « professionnel salarié associatif » une sorte de garantie d'un niveau de reconnaissance élevé.

- le «professionnel méconnu » exerce une activité nouvelle, portée par le secteur associatif. Cependant, elle n'est pas (encore) sanctionnée comme profession et ne correspond pas non plus à une qualification salariale. Le processus de professionnalisation est pourtant engagé par le monde associatif, ne serait-ce que par l'attention portée à la formation ou par les tentatives de structuration de l'activité. Mais, soit que ces prémisses trouvent trop peu d'écho du côté des institutions publiques, soit que le secteur est trop marginal ou isolé pour porter ce processus hors du milieu, soit que l'activité est encore en émergence, le processus n'a pas encore réussi à franchir les limites du monde associatif. Là encore, il s'agit le plus souvent de personnel salarié, mais un bénévole peut également assurer une telle activité ; c'est bien la spécificité du milieu associatif. Nous renvoyons ici à l'exemple évoqué plus haut des « responsables de secteur » dans l'aide à domicile.

Les conseillères conjugales et familiales œuvrant au sein des associations de défense des droits des femmes constituent une autre illustration de ces activités ne bénéficiant que d'une reconnaissance limitée, strictement interne au milieu associatif. La professionnalisation de cette activité est portée par des associations aux orientations très différentes. Elles concernent aussi bien la défense des droits des femmes que le familialisme chrétien, ce qui constitue certainement un handicap dans la réussite du processus (Philippe, 2007).

\footnotetext{
18 Le GIP Aten est l'Atelier technique des espaces naturels, un groupement d'intérêt public créé en 1997. Il regroupe des acteurs publics (ministère de l'Écologie et du Développement durable, Parcs nationaux, Conservatoire de l'espace littoral et des rivages lacustres), associatifs (Réserves naturelles de France, Parcs naturels régionaux, Conservatoires d'espaces naturels) et privés (Station biologique de la tour du Valat).
} 
Là encore, comme pour le travailleur abstrait, la présence possible de bénévoles occupant les mêmes fonctions et l'absence de reconnaissance de ces activités dans le monde salarial font courir le risque d'une dénégation des qualifications pourtant exigées à l'embauche. En l'absence de financements garantis et d'assurance sur la pérennité des structures, les salariées de nombreux CIDFF, par exemple, sont ainsi faiblement rémunérées ; elles sont de fait classées dans les « bas salaires $»^{19}$, alors même que, pour certains postes, un niveau master est systématiquement requis.

La qualité des emplois associatifs apparaît liée non seulement à la position économique des organisations, qui s'exprime plutôt en termes de reconnaissance salariale, mais également à la réussite plus ou moins grande de leurs stratégies de professionnalisation, que l'on pourrait associer à leur positionnement dans l'ordre politique.

Ainsi la faible reconnaissance salariale dont bénéficient les «professionnels méconnus » traduit-elle le plus souvent une situation financière précaire des organisations associatives. Cette précarité les conduisant souvent aussi à reconnaître de façon insuffisante le travail des « salariés professionnels », pourtant mieux protégés par les accords salariaux existant à l'extérieur des associations. Les associations jouent alors le rôle de «mauvais employeurs », orientant à la baisse les rémunérations et faisant de l'emploi associatif un mode déprécié (Hély, 2009). Cette tendance peut être compensée par une reconnaissance d'ordre professionnel, mais c'est alors la force du positionnement politique des associations qui s'exprime ; et ce à travers leur capacité à créer un rapport de forces leur permettant de peser sur les décisions des pouvoirs publics, à la fois en termes de réglementation et de financement.

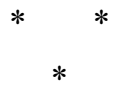

La typologie présentée ici décline les types de travailleurs associatifs (le travailleur abstrait, le professionnel méconnu, le salarié professionnel, le

19 Un salarié perçoit un «bas revenu salarial » si l'ensemble des rémunérations liées à son travail (salaires, primes, indemnités chômage) est inférieur aux deux tiers du salaire médian de l'ensemble de la population (Insee). professionnel associatif salarié) en fonction des modes de reconnaissance qui leur sont accordés. Elle reste à discuter et à affiner. Mais, de notre point de vue, en dissociant les deux processus de salarisation et de professionnalisation, elle permet d'analyser plus finement les risques d'installation durable de mauvaises conditions d'emploi dans le monde associatif. Elle permet aussi de comprendre pourquoi les tentatives des organisations concernées pour professionnaliser leur activité ne débouchent pas forcément sur une meilleure reconnaissance du travail effectué en leur sein. Autrement dit, la professionnalisation de l'activité ne va pas toujours de pair avec la professionnalisation des salariés.

Les conditions de reconnaissance salariale dépendent assurément de la situation économique de l'association. Ainsi, notre typologie montre en particulier que les conditions de cette reconnaissance ne peuvent être déconnectées de la capacité de l'organisation à faire reconnaître plus globalement, par l'ensemble social, la spécificité du travail effectué dans ce cadre comme professionnel. C'est alors la dimension politique de ces organisations aux multiples facettes qui est mobilisée.

Mais cette typologie souligne également combien cette dimension politique, propice à l'engagement désintéressé, peut contribuer à l'instrumentalisation de ces organisations ; instrumentalisation qui se traduit par l'élaboration et la légitimation de normes dégradées d'emploi, à travers une moindre reconnaissance matérielle du travail. À cet égard, la proximité du travail effectué par les salariés associatifs avec le travail bénévole joue un rôle ambigu : d'un côté, elle met en lumière la dimension d'engagement personnel de ce travail, à laquelle peut alors être associée une reconnaissance de type professionnel; mais d'un autre côté, elle tend à en déprécier les aspects les plus objectivables, mieux pris en compte lorsqu'un rapport de forces strictement salarial parvient à s'instaurer.

Dans un contexte économique général de précarisation de l'emploi, beaucoup d'associations apparaissent en porte-à-faux. D'une part, entre leur volonté de développer des services dédiés à l'intérêt général et d'autre part, leur difficulté à se positionner dans les rapports de force caractéristiques du salariat. Leur rapport à la puissance publique est sans doute à questionner dans une période de désengagement de l'État. Ce 
désengagement se traduisant à la fois par la diminution de l'emploi public et par le développement de modes de financement qui font une large place à la concurrence. Loin d'être une simple opération technique, la contractualisation avec les pouvoirs publics peut entraîner les organisations, de plus en plus souvent financées « au projet », mais employeuses de salariés en CDI (contrat à durée indéterminée), dans des déséquilibres périlleux pour la pérennité et pour la qualité de leurs emplois salariés. L'accès à une reconnaissance sociale du travail associatif, bénévole ou salarié, rend ainsi plus que jamais nécessaire l'intégration, par les associations, de leur fonction économique d'employeur au niveau même que leur projet politique.

\section{Bibliographie}

Aballéa F. (2005), « La professionnalisation inachevée des assistantes maternelles », Recherches et Prévisions, $\mathrm{n}^{\circ} 80$, pp. 55-65.

Aballéa F. (2007), « L'échec de l'action publique à professionnaliser les occupations : l'exemple des assistantes maternelles », Journées Internationales de Sociologie du travail (JIST), « Restructurations, précarisation et valeurs », Londres, 20-22 juin 2007.

Castel R. (1995), Les métamorphoses de la question sociale : une chronique du salariat, Paris, Fayard.

Clergeau C., Dussuet A. (2005), « La professionnalisation dans les services à domicile aux personnes âgées: l'enjeu du diplôme », Formation Emploi, $\mathrm{n}^{\circ} 90$, pp. 65-78.

Dussuet A., Flahault E., Loiseau D. (2007), Quelle gestion des ressources humaines dans l'économie sociale? Entre bénévolat et professionnalisation : la place du travail dans les associations, DIIESES.

Dussuet A., Loiseau D. (2007), « Les services aux familles offerts par les associations : un modèle de service "entre" formel et informel ", L'économie sociale entre informel et formel: paradoxes et innovations, Dussuet A., Lauzanas J.-M., Rennes, Presses universitaires de Rennes, coll. « Économie et société », pp. 95-128.

Flahault E., Robic P. (2007), « Les associations d'environnement partenaires privilégiés des pouvoirs publics : un paradoxe qui dynamise la gestion de l'environnement », L'économie sociale entre informel et formel. Paradoxes et innovations, A. Dussuet, J.-M. Lauzanas (dir.), Rennes, PUR, coll. « Économie et société », pp. 183-198.

Fonjep / Ithaque (2003), L'emploi et la fonction employeur dans les associations, http://www.fonjep. org/detail/page_detail_def.aspx?theme $=3$.

Hély M. (2007), « A travail égal, salaire inégal : ce que travailler dans le secteur associatif veut dire », Communication aux JIST, Londres.

Hély M. (2009), Les métamorphoses du monde associatif, Paris, PUF, coll. « Le lien social ».

Honneth A. (2002 [1992]), La lutte pour la reconnaissance, Paris, Cerf.

Hughes E.C. (1996), Le regard sociologique, Paris, Éditions de l'EHESS.

Jany-Catrice F., Puissant E., Ribault T. (2009) " Associations d'aide à domicile : pluralité des héritages, pluralité des professionnalités », Formation Emploi, n 107, pp. 77-91.

Lenoir R. (2003), Généalogie de la morale familiale, Paris, Seuil.

Loiseau D. (1996), Femmes et militantismes, Paris, L'Harmattan. 
Legros D., Narcy M. (2004), « Différentiel de salaire entre secteur sans but lucratif et secteur privé en France », Solidarités collectives - Familles et solidarités, Girard D. (dir.), Paris, L'Harmattan. pp. 215-230.

Marx K. (1975 [1867]), Le capital, livre premier, tome 1, Éditions Sociales.

Naville P. (1956), Essai sur la qualification du travail, Paris, M. Rivière.

Philippe C. (2007), « Le conseil conjugal et familial. Une profession à la recherche de reconnaissance et d'unité », Recherches et prévisions, n 89, pp. 59-63.
Saglio J. (1998), « Qualifications et classifications », Le monde du travail, Kergoat J., Boutet J., Jacot H., Linhart D., Paris, La Découverte, pp. 257-264.

Simonet M. (2006), « Le monde associatif: entre travail et engagement », Sociologie du monde du travail, Norbert Alter (dir.), Paris, PUF, pp. 191-207.

Tchernonog V. (2007), Le paysage associatiffrançais. Mesures et évolutions, Juris-Associations-Dalloz.

Voswinkel S. (2007), « L'admiration sans appréciation. Les paradoxes de la double reconnaissance du travail subjectivisé », Travailler, $\mathrm{n}^{\circ} 18, \mathrm{pp}$. 59-87.

\section{Résumé}

\section{Entre professionnalisation et salarisation, quelle reconnaissance du travail dans le monde associatif? \\ Annie Dussuet, Erika Flahault}

À partir de plusieurs recherches empiriques sur la gestion du travail dans les associations, nous montrerons que leur caractère non lucratif et la coexistence entre travail bénévole et travail salarié induisent certaines particularités. Nous distinguerons, d'une part, le processus de salarisation, qui installe l'organisation dans la position d'employeur de salariés, et d'autre part, la professionnalisation, avec laquelle elle est souvent confondue, en mettant l'accent sur la notion de reconnaissance. Nous proposerons ensuite une typologie des formes de reconnaissance accordée au travail dans les associations, en croisant ces deux dimensions de la salarisation et de la professionnalisation.

Mots clés

Mouvement associatif, professionnalisation, bénévolat, travail salarié

Journal of Economic Literature : J 44 